\title{
SUFFICIENT CONDITIONS FOR INFINITE-HORIZON CALCULUS OF VARIATIONS PROBLEMS
}

\author{
Jö̈L BLOT ${ }^{1}$ AND NAÏLA HAYEK ${ }^{2}$
}

\begin{abstract}
After a brief survey of the literature about sufficient conditions, we give different sufficient conditions of optimality for infinite-horizon calculus of variations problems in the general (non concave) case. Some sufficient conditions are obtained by extending to the infinite-horizon setting the techniques of extremal fields. Others are obtained in a special case of reduction to finite horizon. The last result uses auxiliary functions. We treat five notions of optimality. Our problems are essentially motivated by macroeconomic optimal growth models.
\end{abstract}

AMS Subject Classification. 90A16, 49K99.

Received October 29, 1998. Revised December 9, 1999 and April 17, 2000.

\section{INTRODUCTION}

We consider the following infinite-horizon calculus of variations problems

$$
\text { Maximize } J(x):=\int_{0}^{+\infty} L(t, x(t), \dot{x}(t)) d t \text { with } x(0) \text { given. }
$$

We also study other infinite-horizon optimality notions (Sect. 1).

In the classical finite-horizon setting, there exist two notions of local optimal solution: the strong one and the weak one. The curve $\hat{x}$ is a local strong (respectively weak) solution on a bounded interval $[a, b]$ when it is better than each admissible curve $x$ such that $\|x(t)-\hat{x}(t)\|<c$ (respectively, $\|x(t)-\hat{x}(t)\|+\|\dot{x}(t)-\dot{\hat{x}}(t)\|<c)$ for every $t \in[a, b]$, where the radius $c$ is constant (independent of $t$ ).

In this infinite-horizon setting, following [5], we consider two notions of local optimal solution in a moving radius neighbourhood: $\|x(t)-\hat{x}(t)\|<r(t)$ (respectively $\|x(t)-\hat{x}(t)\|+\|\dot{x}(t)-\dot{\hat{x}}(t)\|<r(t)$ ) for every $t \in \mathbb{R}_{+}$.

The Euler-Lagrange equation associated to the functional $J$ is

$$
L_{x}(t, x(t), \dot{x}(t))=\frac{d}{d t} L_{\dot{x}}(t, x(t), \dot{x}(t))
$$

A solution of this equation, defined on an interval $\mathcal{I}$, is called an extremal on $\mathcal{I}$.

Keywords and phrases: Calculus of variations, infinite-horizon problems, optimal growth theory.

1 CERMSEM, M.S.E., Université de Paris 1 Panthéon-Sorbonne, 106-112 boulevard de l'Hôpital, 75647 Paris Cedex 13, France; e-mail: blot@univ-paris1.fr

2 L.I.B.R.E., Faculté de Droit et des Sciences Économiques, Université de Franche-Comté, avenue de l'Observatoire, 25030 Besançon Cedex, France, and CERMSEM, M.S.E., Université de Paris 1 Panthéon - Sorbonne, 106-112 boulevard de l'Hôpital, 75647 Paris Cedex 13, France; e-mail: hayek@univ-paris1.fr 
In [4], we have studied necessary conditions, notably second-order conditions. In the present work, we want to build sufficient conditions to ensure that an extremal is optimal.

We briefly describe the contents of the paper.

In Section 1, we precise our framework. In Section 2, we precise several notions associated to an extremal, especially the notion of conjugate point. In Section 3, we give a list of assumptions along an extremal. In Section 4, we present a brief survey of the literature on sufficient conditions for optimal control and calculus of variations problems, in the finite and infinite-horizon cases.

In the following sections we present our new results. In Section 5, we establish sufficient conditions for an extremal to be a local optimal solution in a moving radius neighbourhood. In the literature on sufficient conditions, the most usual (sufficient) transversality condition at infinity is the Seierstad-Sydsaeter condition (cf. Sect. 3) which only works under global concavity assumptions. To treat nonconcave cases, we need to extend to infinite-horizon some tools related to extremal fields (which are classical in finite-horizon) and we use a transversality condition at infinity too, denoted by (E,x) in Section 4. In Section 6, we give sufficient conditions in a special case where the infinite-horizon problem can be transformed into a finite-horizon problem. We establish sufficient conditions for an extremal to be a local optimal solution in a constant radius neighbourhood. Finally in Section 7, we give sufficient conditions via auxiliary functions.

In Appendix, we precise the notion of (Mayer) extremal field that we use in the infinite-horizon framework and we establish several results to ensure that an extremal curve is embeddable in an extremal field. $[31]$.

These problems are motivated by macroeconomic optimal growth models, and by several physics problems

\section{Framework AND NOtions of optimality}

Let $\mathcal{O}$ be a nonempty open subset of $\mathbb{R} \times \mathbb{R}^{n} \times \mathbb{R}^{n}$ such that $\operatorname{proj}_{1}(\mathcal{O}) \supset \mathbb{R}_{+}$. We can consider $\mathcal{O}$ as the graph of a correspondence from $\mathbb{R} \times \mathbb{R}^{n}$ in $\mathbb{R}^{n}$, and so, for each $(t, x) \in \mathbb{R} \times \mathbb{R}^{n}$, we set

$$
\begin{aligned}
\mathcal{O}(t, x) & :=\left\{v \in \mathbb{R}^{n}:(t, x, v) \in \mathcal{O}\right\}, \text { and } \\
D(\mathcal{O}) & :=\left\{(t, x) \in \mathbb{R} \times \mathbb{R}^{n}: \mathcal{O} \neq \emptyset\right\} .
\end{aligned}
$$

We always assume that, for every $(t, x) \in D(\mathcal{O}), \mathcal{O}(t, x)$ is convex.

When $f$ is a function, we denote by $\mathcal{G}(f)$ its graph. We consider the following functions space:

$$
C_{\mathcal{O}}^{1}:=\left\{x \in C^{1}\left(\mathbb{R}_{+}, \mathbb{R}^{n}\right): \mathcal{G}(x, \dot{x}) \subset \mathcal{O}\right\}
$$

We shall assume that this functions space is nonempty.

Let $L \in C^{0}(\mathcal{O}, \mathbb{R})$ be a Lagrangian function. We consider the two following conditions:

$$
\begin{aligned}
& L \in C^{1}(\mathcal{O}, \mathbb{R}) \\
& L \in C^{2}(\mathcal{O}, \mathbb{R}) .
\end{aligned}
$$

For every $T \in(0,+\infty)$, and, for every $x \in C_{\mathcal{O}}^{1}$, we define the functional

$$
J_{T}(x):=\int_{0}^{T} L(t, x(t), \dot{x}(t)) d t
$$

and we denote by $\operatorname{dom}(J)$ the set of paths $x \in C_{\mathcal{O}}^{1}$ such that the following improper Riemann integral exists in $\mathbb{R}$ :

$$
J(x)=\lim _{T \rightarrow+\infty} J_{T}(x)=\int_{0}^{+\infty} L(t, x(t), \dot{x}(t)) d t .
$$


We fix $\eta \in \mathbb{R}^{n}$ such that $\mathcal{O}(0, \eta)$ is nonempty, and we set

$$
\mathcal{A} \operatorname{dm}(\eta):=\{x \in \operatorname{dom}(J): x(0)=\eta\} .
$$

We shall assume that $\mathcal{A} \mathrm{dm}(\eta)$ is nonempty.

We now give different nonequivalent notions of optimality following the framework of [3-5] for the first one, and the notions provided in [7] for the others.

Definitions 1. Let $x \in C_{\mathcal{O}}^{1}$ and $y \in C_{\mathcal{O}}^{1}$ satisfy $x(0)=y(0)=\eta$. We say that:

(I) $x$ is (I)-greater than $y$, when $x, y \in \mathcal{A d m}(\eta)$, and $J(y) \leq J(x)$.

(II) $x$ is (II)-greater than $y$, when $x \in \mathcal{A d m}(\eta)$, and $\limsup _{T \rightarrow+\infty} J_{T}(y) \leq J(x)$.

(III) $x$ is (III)-greater than $y$, when $\lim \sup \left(J_{T}(y)-J_{T}(x)\right) \leq 0$.

(IV) $x$ is (IV)-greater than $y$, when $\liminf _{T \rightarrow+\infty}\left(J_{T}(y)-J_{T}(x)\right) \leq 0$.

(V) $x$ is $(\mathrm{V})$-greater than $y$, when for every $T \in(0,+\infty)$ such that $y(T)=x(T)$, we have $J_{T}(y) \leq J_{T}(x)$.

Definitions 2. Let $Z \in\{I, I I, I I I, I V, V\}$. Let $x \in C_{\mathcal{O}}^{1}$ satisfy $x(0)=\eta$. When $Z \in\{I, I I\}$, we assume moreover that $x \in \mathcal{A d m}(\eta)$. The path $x$ is called:

(i) (Z)-optimal, when for each $y \in C_{\mathcal{O}}^{1}$ such that $y(0)=\eta$ (and such that $y \in \mathcal{A d m}(\eta)$ if $\mathrm{Z}=\mathrm{I}$ ), $x$ is (Z)-greater than $y$.

(ii) locally strong (Z)-optimal, when there exists $r \in C^{0}\left(\mathbb{R}_{+},(0,+\infty)\right)$ such that for every $y \in C_{\mathcal{O}}^{1}$ verifying $y(0)=\eta$ (and verifying $y \in \mathcal{A} \operatorname{dm}(\eta)$ if $\mathrm{Z}=\mathrm{I}$ ), we have: $\left[\forall t \in \mathbb{R}_{+},\|y(t)-x(t)\|<r(t)\right]$ implies that $x$ is (Z)-greater than $y$.

(iii) locally weak (Z)-optimal, when there exists $r \in C^{0}\left(\mathbb{R}_{+},(0,+\infty)\right)$ such that for every $y \in C_{\mathcal{O}}^{1}$ verifying $y(0)=\eta$ (and verifying $y \in \mathcal{A d m}(\eta)$ if $\mathrm{Z}=\mathrm{I}$ ), we have: $\left[\forall t \in \mathbb{R}_{+},\|y(t)-x(t)\|+\|\dot{y}(t)-\dot{x}(t)\|<r(t)\right]$ implies that $x$ is (Z)-greater than $y$.

The relationship between these notions of optimality is the following:

$$
\begin{aligned}
{[x(\mathrm{II})-\text { optimal }] } & \Longrightarrow[x(\mathrm{III})-\text { optimal }] \Longrightarrow[x(\mathrm{IV})-\text { optimal }] \\
& \Longrightarrow[x(\mathrm{I})-\text { optimal }] \Longrightarrow[x(\mathrm{~V})-\text { optimal }]
\end{aligned}
$$

and similar implications hold for locally strong and locally weak notions of optimality. The first three implications are easy to prove. To prove the last ones, we need a Rounding-Off Corners theorem $[3,5]$.

\section{Notions Associated to An EXtremal}

The Hamiltonian associated to the Lagrangian $L$ [5] is the function

$$
\begin{gathered}
H: D(\mathcal{O}) \times \mathbb{R}^{n} \longrightarrow(-\infty,+\infty] \quad \text { defined by } \\
H(t, x, p):=\sup \{p \cdot v+L(t, x, v): v \in \mathcal{O}(t, x)\} .
\end{gathered}
$$

We consider the following condition of finiteness and smoothness on $H$ :

$$
H \in C^{2}(\mathcal{O}, \mathbb{R}) .
$$

A sufficient condition to ensure (4) is the following:

$$
\exists c<0, \forall(t, x, v) \in \mathcal{O}, \forall w \in \mathbb{R}^{n}, \quad L_{\dot{x} \dot{x}}(t, x, v)(w, w) \leq c\|w\|^{2} .
$$


Under this condition the Hamiltonian system associated to (1) is:

$$
\left.\begin{array}{rl}
\dot{x} & =H_{p}(t, x, p) \\
\dot{p} & =-H_{x}(t, x, p)
\end{array}\right\}
$$

We denote by $\left(\mathrm{X}\left(t, t_{0}, x_{0}, \alpha\right), \mathrm{P}\left(t, t_{0}, x_{0}, \alpha\right)\right)$ the flow of $(5)$. When $x$ is an extremal on $\mathcal{I}$, we recall the Jacobi equation along $x$ :

$$
L_{x x} h+L_{x \dot{x}} \dot{h}=\frac{d}{d t}\left[L_{\dot{x} x} h+L_{\dot{x} \dot{x}} \dot{h}\right]
$$

where the arguments of the second partial differentials of $L$ are taken at $(t, x(t), \dot{x}(t))$.

Let $\hat{x}$ be an extremal on an unbounded from above interval $\mathcal{I}$. For each $t_{0} \in \mathcal{I}$, we consider the subset $\mathcal{A}\left(t_{0}, \hat{x}\right)$ of $\mathbb{R}^{n}$ constituted by the $\alpha \in \mathbb{R}^{n}$ such that $\left(\mathrm{X}\left(t, t_{0}, \hat{x}\left(t_{0}\right), \alpha\right), \mathrm{P}\left(t, t_{0}, \hat{x}\left(t_{0}\right), \alpha\right)\right)$ is defined for all $t \in\left[t_{0},+\infty\right)$. Setting $\hat{p}(t):=-L_{\dot{x}}(t, \hat{x}(t), \dot{\hat{x}}(t))$, we notice that $\mathcal{A}\left(t_{0}, \hat{x}\right)$ is nonempty since it contains $\hat{p}\left(t_{0}\right)$.

We assume fulfilled the following condition:

$$
\text { for every } t_{0} \in \mathcal{I}, \mathcal{A}\left(t_{0}, \hat{x}\right) \text { is open in } \mathbb{R}^{n} \text {. }
$$

We define the family $\left(\Phi\left(., t_{0}, \alpha\right), \Psi\left(., t_{0}, \alpha\right)\right)_{\alpha \in \mathcal{A}\left(t_{0}, \hat{x}\right)}$ as follows:

$$
\left.\begin{array}{l}
\Phi\left(t, t_{0}, \alpha\right):=\mathrm{X}\left(t, t_{0}, \hat{x}\left(t_{0}\right), \alpha\right) \\
\Psi\left(t, t_{0}, \alpha\right):=\mathrm{P}\left(t, t_{0}, \hat{x}\left(t_{0}\right), \alpha\right)
\end{array}\right\}
$$

We note that $\Phi\left(t_{0}, t_{0}, \alpha\right)=\hat{x}\left(t_{0}\right), \Psi\left(t_{0}, t_{0}, \alpha\right)=\alpha, \Phi\left(t, t_{0}, \hat{p}\left(t_{0}\right)\right)=\hat{x}(t)$ and $\Psi\left(t, t_{0}, \hat{p}\left(t_{0}\right)\right)=\hat{p}(t)$ for every $t \in\left[t_{0},+\infty\right)$.

We recall the following definition [4].

Definition 3. Under (3), let $\hat{x}$ be an extremal on $\left[t_{0},+\infty\right), t_{0} \in \operatorname{proj}_{1}(\mathcal{O})$, and $\tau \in\left(t_{0},+\infty\right)$. We say that $\tau$ is a conjugate point to $t_{0}$ (with respect to $\hat{x}$ ) when there exists a solution $h$ of the Jacobi equation along $\hat{x}$ on $\left[t_{0},+\infty\right)$ such that $h\left(t_{0}\right)=h(\tau)=0$ and $L_{\dot{x} \dot{x}}(\tau, x(\tau) \dot{x}(\tau)) \dot{h}(\tau) \neq 0$.

Under (4), $\tau$ is a conjugate point to $t_{0}$ (with respect to $\hat{x}$ ) if and only if: $\operatorname{det} \Phi_{\alpha}\left(\tau, \hat{p}\left(t_{0}\right)\right)=0$. We introduce the following definition:

Definition 4. Under (3), let $\hat{x}$ be an extremal on $\left[t_{0},+\infty\right), t_{0} \in \operatorname{proj}_{1}(\mathcal{O})$, and $\tau \in\left(t_{0},+\infty\right)$. We say that $+\infty$ is conjugate to $\tau$ (with respect to $\hat{x}$ ) when there exists a nontrivial solution $h$ of the Jacobi equation along $\hat{x}$ on $\left[t_{0},+\infty\right)$ such that $\lim _{t \rightarrow+\infty} h(t)=h(\tau)=0$.

We recall that the Weierstrass excess function is defined by:

$$
\mathcal{E}(t, x, v, w):=L(t, x, w)-L(t, x, v)-L_{\dot{x}}(t, x, v) .(w-v)
$$

The domain of definition of $\mathcal{E}$ is

$$
\left\{(t, x, v, w) \in \mathbb{R} \times \mathbb{R}^{n} \times \mathbb{R}^{n} \times \mathbb{R}^{n}:(t, x, v) \in \mathcal{O},(t, x, w) \in \mathcal{O}\right\}
$$




\section{A BRIEF SURVEY OF SUFFICIENT CONDITIONS}

For finite-horizon optimal control problems, we follow Seierstad and Sydsaeter [25] and Hartl et al. [16], to enumerate the known results. Mangasarian [20] proved that the necessary conditions together with the concavity of the Hamiltonian function with respect to the state and control variables were sufficient for optimality. Later, Arrow [2] proposed a result where concavity, with respect to the state variable, of the Hamiltonian maximized with respect to the control variable, replaced Mangasarian concavity of the Hamiltonian. Leitman and Stalford [18], then Seierstad and Sydsaeter [25] gave sufficient conditions where they weakened Arrow's condition. We can consider these conditions as "almost concavity" conditions.

For infinite-horizon optimal control problems, the previous results were extended by adding transversality conditions at infinity of the form $\limsup _{T \rightarrow+\infty} \lambda(T)(x(T)-\hat{x}(T)) \geq 0$ or $\liminf _{T \rightarrow+\infty} \lambda(T)(x(T)-\hat{x}(T)) \geq 0$, for all admissible $x$, where $\lambda$ is the adjoint function.

Recall that the first works on infinite horizon are due to Pontryagin and his school [22], where they briefly discuss infinite horizon optimal control problems in the special case of bounded controls and with the condition that the state has a given limit at infinity.

Other viewpoints are found for instance in Zeidan [30], Sorger [28], where a Riccati matrix differential inequality is used.

We cite also the method of regular synthesis of Boltyanskii [9]. We will not discuss dynamic programming and rather send the reader to Fleming and Rishel [12] and Fleming and Soner [13].

For finite-horizon calculus of variations problems, sufficient conditions under concavity of the Lagrangian are found in [29] (p. 208) whereas sufficient conditions without concavity assumptions are based on conjugate points, Jacobi condition and extremal fields $[1,11,14,17,23], \ldots$

For infinite-horizon calculus of variations problems, we shall give the following theorems adapted from control theory:

Theorem 1. Under (2), let $\hat{x} \in C_{\mathcal{O}}^{1}$ satisfy $\hat{x}(0)=\eta$. If the following conditions are fulfilled:

- $\hat{x}$ satisfies the Euler-Lagrange equation;

- $L$ is concave with respect to $(x, \dot{x})$;

- $\limsup _{T \rightarrow+\infty} L_{\dot{x}}(T, \hat{x}(T), \dot{\hat{x}}(T))(x(T)-\hat{x}(T)) \leq 0$, for all $x \in C_{\mathcal{O}}^{1}$ such that $\hat{x}(0)=\eta$

then $\hat{x}$ is (III)-optimal.

One could take $\liminf _{T \rightarrow+\infty} L_{\dot{x}}(T, \hat{x}(T), \dot{\hat{x}}(T))(x(T)-\hat{x}(T)) \leq 0$ and get $\hat{x}$ is (IV)-optimal.

Theorem 2. Under (2), let $\hat{x} \in C_{\mathcal{O}}^{1}$ satisfy $\hat{x}(0)=\eta$. If the following conditions are fulfilled:

- $\hat{x}$ satisfies the Euler-Lagrange equation;

- $\mathcal{E}(t, \hat{x}, \dot{\hat{x}}, z) \leq 0$ for all $z \in \mathcal{O}(t, \hat{x})$;

- $\left[x \rightarrow H\left(t, x,-L_{\dot{x}}(t, \hat{x}(t), \dot{\hat{x}}(t))\right)\right]$ is concave;

- $\limsup _{T \rightarrow+\infty} L_{\dot{x}}(T, \hat{x}(T), \dot{\hat{x}}(T))(x(T)-\hat{x}(T)) \leq 0$ for all $x \in C_{\mathcal{O}}^{1}$ such that $\hat{x}(0)=\eta$

then $\hat{x}$ is (III)-optimal.

Theorem 3. Under (2), let $\hat{x} \in C_{\mathcal{O}}^{1}$ satisfy $\hat{x}(0)=\eta$. If the following conditions are fulfilled:

- $\hat{x}$ satisfies the Euler-Lagrange equation;

- for all $x \in C_{\mathcal{O}}^{1}$ such that $\hat{x}(0)=\eta$ we have $L(t, \hat{x}(t), \dot{\hat{x}}(t))-L(t, x(t), \dot{x}(t)) \geq L_{x}(t, \hat{x}(t), \dot{\hat{x}}(t))(\hat{x}(t)-x(t))$ $+L_{\dot{x}}(t, \hat{x}(t), \dot{\hat{x}}(t))(\dot{\hat{x}}(t)-\dot{x}(t))$; 
- $\limsup _{T \rightarrow+\infty} L_{\dot{x}}(T, \hat{x}(T), \dot{\hat{x}}(T))(x(T)-\hat{x}(T)) \leq 0$, for all $x \in C_{\mathcal{O}}^{1}$ such that $\hat{x}(0)=\eta$

then $\hat{x}$ is (III)-optimal.

Theorem 4. Under (2), let $\hat{x} \in C_{\mathcal{O}}^{1}$ satisfy $\hat{x}(0)=\eta$. If the following conditions are fulfilled:

- $\hat{x}$ satisfies the Euler-Lagrange equation;

- $\mathcal{E}(t, \hat{x}, \dot{\hat{x}}, z) \leq 0$ for all $z \in \mathcal{O}(t, \hat{x})$;

- for all $x \in C_{\mathcal{O}}^{1}$ such that $\hat{x}(0)=\eta$ we have $H\left(t, x(t),-L_{\dot{x}}(t, \hat{x}(t), \dot{\hat{x}}(t))\right)-H\left(t, \hat{x}(t),-L_{\dot{x}}(t, \hat{x}(t), \dot{\hat{x}}(t))\right)$ $\leq L_{x}(t, \hat{x}(t), \dot{\hat{x}}(t))(x(t)-\hat{x}(t))$

- $\limsup _{T \rightarrow+\infty} L_{\dot{x}}(T, \hat{x}(T), \dot{\hat{x}}(T))(x(T)-\hat{x}(T)) \leq 0$ for all $x \in C_{\mathcal{O}}^{1}$ such that $\hat{x}(0)=\eta$

then $\hat{x}$ is (III)-optimal.

\section{The Assumptions}

We denote by $B C^{0}\left(\mathbb{R}_{+},(0,+\infty)\right)$ the space of continuous bounded functions from $\mathbb{R}_{+}$in $(0,+\infty)$. Under (3), when $x \in C_{\mathcal{O}}^{1}$, we consider the following conditions:

$(\mathbf{A}, x): x$ is an extremal.

$(\mathbf{B}, x):$ There exists $r \in B C^{0}\left(\mathbb{R}_{+},(0,+\infty)\right)$ such that for every $(t, y, v, w)$ verifying $(t, y, v) \in \mathcal{O},(t, y, w) \in \mathcal{O}$, and $\|y-x(t)\|+\|v-\dot{x}(t)\|<r(t)$, we have $\mathcal{E}(t, y, v, w) \leq 0$.

$\left(\mathbf{B}^{\prime}, x\right)$ : There exists $c_{1}>0$ such that for every $(t, y, v, w)$ verifying $(t, y, v) \in \mathcal{O},(t, y, w) \in \mathcal{O}$, and $\|y-x(t)\|+\left\|v-e^{\delta t} \dot{x}(t)\right\|<c_{1}$, we have $\mathcal{E}\left(t, y, e^{-\delta t} v, e^{-\delta t} w\right) \leq 0$.

$(\mathbf{C}, x)$ : For every $t \in \mathbb{R}_{+}$, we have $L_{\dot{x} \dot{x}}(t, x(t), \dot{x}(t))<0$ (negative definite) (the strengthened Legendre condition).

$\left(\mathbf{C}^{\prime}, x\right)$ : There exists $r \in B C^{0}\left(\mathbb{R}_{+},(0,+\infty)\right)$ such that for every $(t, y, v) \in \mathcal{O}$ verifying $\|y-x(t)\|+\|v-\dot{x}(t)\|$ $<r(t)$, we have $L_{\dot{x} \dot{x}}(t, y, v) \leq 0$ (negative semidefinite).

(C", $x)$ : For every $t \in \mathbb{R}_{+}$, we have $L_{\dot{x} \dot{x}}(t, x(t), \dot{x}(t)) \leq 0$ (negative semidefinite) (the Legendre condition).

$(\mathbf{D}, x)$ : There does not exist any conjugate point to 0 (with respect to $x$ ) in $(0,+\infty)$ (Jacobi condition).

(D', $x)$ : There exists $t_{0} \in(-\infty, 0)$ such that $x$ is extendable (as an extremal) to $\left[t_{0},+\infty\right)$ and there does not exist any point in $\left[t_{0}, 0\right)$ which is conjugate to a point of $(0,+\infty)$ (with respect to $x$ ).

$(\mathbf{D},, x)$ : There does not exist any conjugate point to 0 (with respect to $x$ ) in $(0,+\infty)$ and $+\infty$ is not conjugate to 0 ("Strong" Jacobi condition).

$(\mathbf{E}, x): \lim _{t \rightarrow+\infty} L_{\dot{x}}(t, x(t), \dot{x}(t))=0$.

In $[4,5]$, it is shown that $(\mathrm{A}, x),\left(\mathrm{C}^{\prime \prime}, x\right),(\mathrm{D}, x)$ are necessary conditions of $Z$-optimality, for all $Z$.

Except the last three conditions, these conditions are similar to the classical ones for finite-horizon calculus of variations problems $[1,11,14,23]$.

Since $\mathcal{O}$ is open and since $\operatorname{proj}_{1}$ is an open mapping $\left([27]\right.$, p. 63), $\operatorname{proj}_{1}(\mathcal{O})$ is open in $\mathbb{R}$ and it contains $\mathbb{R}_{+} ;$and so there exists $\sigma<0$ such that $\operatorname{proj}_{1}(\mathcal{O}) \supset(\sigma,+\infty)$. By using (4) and the existence of nonextendable solutions of ODE, since the domain of definition of a nonextendable solution is an open interval [6], $x$ is extendable to an extremal defined on $\left(t_{1},+\infty\right)$, where $t_{1} \in(\sigma, 0)$. This allows us to formulate condition $\left(\mathrm{D}^{\prime}, x\right)$.

We note that in a finite-horizon framework, a condition like $(\mathrm{D}, x)$ implies a condition like $\left(\mathrm{D}^{\prime}, x\right)[1]$, but in our infinite-horizon framework, this implication is not guaranteed.

\section{SufFicient CONDitions VIA EXTREMAL FIELDS}

We provide sufficient conditions by using extremal fields for infinite-horizon problems. Notions about extremal fields (in infinite horizon problems) are given in Appendix. 
Lemma 1. Assume that (3) is satisfied. Let $\hat{x} \in C_{\mathcal{O}}^{1}$. If $(C, \hat{x})$ is satisfied then there exists $s \in C^{0}\left(\mathbb{R}_{+},(0,+\infty)\right)$ such that for every $y: \mathbb{R}_{+} \longrightarrow \mathbb{R}^{n}$, for every $z: \mathbb{R}_{+} \longrightarrow \mathbb{R}^{n}$ verifying, for every $t \in \mathbb{R}_{+},(t, y(t), z(t)) \in \mathcal{O}$ and $\|y(t)-\hat{x}(t)\|+\|z(t)-\dot{\hat{x}}(t)\|<s(t)$, we have, for every $t \in \mathbb{R}_{+}, L_{\dot{x} \dot{x}}(t, y(t), z(t))<0$ (definite negative).

Proof. For each $n \in \mathbb{N}$, the compactness of $K_{n}:=\{(t, \hat{x}(t), \dot{\hat{x}}(t)): t \in[n, n+1]\}$, ensures the existence of $\rho_{n}>0$, such that $L_{\dot{x} \dot{x}}$ is negative definite on $\left\{(t, y, z): d\left((t, y, z) ; K_{n}\right)<\rho_{n}\right\}$. Proceeding as in the proof of Proposition A1, we obtain $s$.

Lemma 2. Assume that (3) is satisfied. Let $\hat{x} \in C_{\mathcal{O}}^{1}$ satisfy $\hat{x}(0)=\eta$ and be embeddable in a field $f: N \longrightarrow M$. Let $u: M \longrightarrow \mathbb{R}^{n}$ be the slope of $f$.

Then for every $r \in C^{0}\left(\mathbb{R}_{+},(0,+\infty)\right)$, there exists $r_{1} \in C^{0}\left(\mathbb{R}_{+},(0,+\infty)\right)$ such that, for all $x \in C_{\mathcal{O}}^{1}$ verifying for every $t \in \mathbb{R}_{+},(t, x(t)) \in M$, we have: $\left[\forall t \in \mathbb{R}_{+},\|x(t)-\hat{x}(t)\|<r_{1}(t)\right]$ implies $\left[\forall t \in \mathbb{R}_{+},\|x(t)-\hat{x}(t)\|\right.$ $+\|u(t, x(t))-u(t, \hat{x}(t))\|<r(t)]$.

Proof. Let $r \in C^{0}\left(\mathbb{R}_{+},(0,+\infty)\right)$ be given. For each $n \in \mathbb{N}$, we consider $[n, n+1]$. Since $u$ is continuous from $M$ to $\mathbb{R}^{n}$, the Nemytskii operator $\mathcal{N}_{u_{[n, n+1]}}$ is continuous from $C^{0}\left([n, n+1], \mathbb{R}^{n}\right)$ to $C^{0}\left([n, n+1], \mathbb{R}^{n}\right)$. So by taking $\mu_{n}:=\inf r([n, n+1])>0$, we can assert the existence of $\xi_{n}>0, \xi_{n} \leq \frac{\mu_{n}}{2}$ such that, for every $y \in C^{0}\left([n, n+1], \mathbb{R}^{n}\right)$ whose graph is included in $M$, for every $t \in[n, n+1],\|y(t)-\hat{x}(t)\| \leq \xi_{n}$ implies that, for every $t \in[n, n+1],\|u(t, y(t))-u(t, \hat{x}(t))\| \leq \frac{\mu_{n}}{2}$, which implies that for every $t \in[n, n+1]$, $\|y(t)-\hat{x}(t)\|+\|u(t, y(t))-u(t, \hat{x}(t))\| \leq \mu_{n}$. Proceeding as in the proof of Proposition A1, we obtain $r_{1}$.

Proposition 1. Under (3), let $\hat{x} \in C_{\mathcal{O}}^{1}$ satisfy $\hat{x}(0)=\eta$. We assume that the following conditions are fulfilled.

(i) $\hat{x}$ is embeddable in a Mayer field $f: N \longrightarrow M$ whose slope is $u: M \longrightarrow \mathbb{R}^{n}$.

(ii) $(E, \hat{x})$ is satisfied.

(iii) For every $x \in C_{\mathcal{O}}^{1}$ whose graph is included in $M$ and verifying $x(0)=\eta$ (respectively there exists $r \in$ $C^{0}\left(\mathbb{R}_{+},(0,+\infty)\right)$ such that, for every $x \in C_{\mathcal{O}}^{1}$ whose graph is included in $M$ and verifying: $x(0)=\eta$ and for every $\left.t \in \mathbb{R}_{+},\|x(t)-\hat{x}(t)\|+\|\dot{x}(t)-\dot{\hat{x}}(t)\|<r(t)\right)$, we have $\mathcal{E}(t, x(t), u(t, x(t)), \dot{x}(t)) \leq 0$.

Then $\hat{x}$ is local strong (III)-optimal (respectively local weak (III)-optimal).

Moreover if $\hat{x} \in \operatorname{Adm}(\eta)$, then $\hat{x}$ is local strong (II)-optimal (respectively local weak (II)-optimal).

Proof. Under (i) and (ii), Proposition A3 implies the existence of a function $r_{1} \in B C^{0}\left(\mathbb{R}_{+},(0,+\infty)\right)$ such that, for every $x \in C_{\mathcal{O}}^{1}$ whose graph is included in $M$ and verifying: $x(0)=\eta$ and for every $t \in \mathbb{R}_{+},\|x(t)-\hat{x}(t)\|$ $<r_{1}(t)$, we have

$$
\limsup _{T \rightarrow+\infty}\left(J_{T}(x)-J_{T}(\hat{x})\right)=\limsup _{T \rightarrow+\infty} \int_{0}^{T} \mathcal{E}(t, x(t), u(t, x(t)), \dot{x}(t)) d t .
$$

Hence, by (iii), we have for these curves $x$ : $\limsup _{T \rightarrow+\infty}\left(J_{T}(x)-J_{T}(\hat{x})\right) \leq 0$, so the first conclusion follows. The reasoning is similar for the second (local) part of (iii).

Theorem 5. Under (3) and (4), let $\hat{x} \in C_{\mathcal{O}}^{1}$ satisfy $\hat{x}(0)=\eta$. If $(A, \hat{x}),(B, \hat{x})$ or $\left(C^{\prime}, \hat{x}\right),(C, \hat{x}),\left(D^{\prime}, \hat{x}\right)$, $(E, \hat{x})$ (respectively $\left.(A, \hat{x}),(C, \hat{x}),\left(D^{\prime}, \hat{x}\right),(E, \hat{x})\right)$ are fulfilled, then $\hat{x}$ is local strong (III)-optimal (respectively local weak (III)-optimal).

Moreover if $\hat{x} \in \operatorname{Adm}(\eta)$, then $\hat{x}$ is local strong (II)-optimal (respectively local weak (II)-optimal). 
Proof. Since $(\mathrm{A}, \hat{x}),(\mathrm{C}, \hat{x})$, and (D', $\hat{x})$ are satisfied, Proposition A2 implies that $\hat{x}$ is embeddable in a Mayer field. Since $(\mathrm{E}, \hat{x})$ is satisfied, the conclusion of Proposition 1 holds. Let $r_{1}$ be obtained by that theorem. Let $r$ be the function given by $(\mathrm{B}, \hat{x})$. Lemma 2 implies thus the existence of a function $r_{2} \in B C^{0}\left(\mathbb{R}_{+},(0,+\infty)\right)$ such that, for every $x \in C_{\mathcal{O}}^{1}$ whose graph is included in $M$, we have

$$
\left.\left[\forall t \in \mathbb{R}_{+},\|x(t)-\hat{x}(t)\|<r_{2}(t)\right] \Longrightarrow\left[\forall t \in \mathbb{R}_{+},\|x(t)-\hat{x}(t)\|+\| u(t, x(t))-u(t, \hat{x}(t))\right) \|<r(t)\right] .
$$

Let $r^{*}:=\min \left\{r_{1}, r_{2}\right\} \in B C^{0}\left(\mathbb{R}_{+},(0,+\infty)\right)$, and we have

$$
\left[\forall t \in \mathbb{R}_{+},\|x(t)-\hat{x}(t)\|<r^{*}(t)\right] \Longrightarrow\left[\forall t \in \mathbb{R}_{+}, \mathcal{E}(t, x(t), u(t, x(t)), \dot{x}(t)) \leq 0\right] .
$$

The first part of the conclusion follows from Proposition 1. The reasoning is similar for the second part.

\section{Sufficient CONDItions in A CASE OF REDUCTION TO Finite HORIZON}

The special case $L(t, x, \dot{x})=e^{-\delta t} \ell(x, \dot{x})$ with $\delta>0$, arises in optimal growth macroeconomic theory [19, 24]. From $\Omega$, a nonempty open subset of $\mathbb{R}^{n} \times \mathbb{R}^{n}$, we build $\mathcal{O}:=\mathbb{R}_{+} \times \Omega$.

Theorem 5 can be totally applied in this case. But taking into account the specification of this case, following a reduction to finite-horizon encountered in [3], we can state a result in a neighbourhood of constant radius. For this, we introduce the following function space:

$$
N C_{\mathcal{O}}^{1}:=\left\{x \in C_{\mathcal{O}}^{1}: \lim _{t \rightarrow+\infty} x(t), \lim _{t \rightarrow+\infty} \dot{x}(t) \text { and } \lim _{t \rightarrow+\infty} \dot{x}(t) e^{\delta t} \text { exist in } \mathbb{R}^{n}\right\}
$$

Since $\lim _{t \rightarrow+\infty} x(t)$ is finite, we necessarily have $\lim _{t \rightarrow+\infty} \dot{x}(t)=0$.

We introduce two new assumptions:

$$
\forall x \in C_{\mathcal{O}}^{1}, \forall s \in \mathbb{R}_{+},\{(t, x(s), 0): t \geq s\} \subset \mathcal{O}
$$

$\ell$ is bounded from below on $\Omega$.

By replacing $\ell$ by $\ell-\inf \ell(\Omega)$, we do not change the optimal local solution of the variational problem, and so condition (9) is equivalent to the condition $\ell \geq 0$.

Theorem 6. Under $(3,4,8)$ and (9), let $\hat{x} \in N C_{\mathcal{O}}^{1} \cap \operatorname{Adm}(\eta)$. If $(A, \hat{x}),(B$, $\hat{x}),(C, \hat{x}),(D$ ", $\hat{x})$ are fulfilled then there exists a constant $c>0$ such that for every $z \in \operatorname{Adm}(\eta)$ we have:

$$
\forall t \in \mathbb{R}_{+},\|z(t)-\hat{x}(t)\|<c \Longrightarrow J(z) \leq J(\hat{x})
$$

Proof. Following the method used in [3], we introduce the function $\gamma:[0,+\infty] \rightarrow\left[-\delta^{-1}, 0\right], \gamma(t):=\left(-\delta^{-1}\right) e^{-\delta t}$ and we define the function $y$ by $y(s):=x \circ \gamma^{-1}(s)$ when $s \in\left[-\delta^{-1}, 0\right]$ and $x \in N C_{\mathcal{O}}^{1}$. Thus $y\left(-\delta^{-1}\right)=\eta$ and $y(0)=\lim _{t \rightarrow+\infty} x(t)$ exists by hypothesis.

We consider the following finite-horizon calculus of variations problem:

$$
\left(\mathcal{F}_{\delta}\right) \text { Maximize } \mathcal{J}_{\delta}(y):=\int_{-1 / \delta}^{0} \mathcal{L}(s, y(s), \dot{y}(s)) d s=\int_{-1 / \delta}^{0} \ell(y(s),-\delta . s . \dot{y}(s)) d s
$$


when $y \in C^{1}\left(\left[-\delta^{-1}, 0\right], \mathbb{R}^{n}\right),(y(s),-\delta . s . \dot{y}(s)) \in \Omega, s \in\left[-\delta^{-1}, 0\right], y\left(-\delta^{-1}\right)=\eta$.

We shall first show that $\hat{y}=\hat{x} \circ \gamma^{-1}$ is a local strong solution of this problem, in the usual sense [1]. Since $(\mathrm{A}, \hat{x})$ is verified, $\hat{x}$ satisfies the following Euler-Lagrange equation:

$$
\forall t \in \mathbb{R}, \quad \ell_{x}(\hat{x}(t), \dot{\hat{x}}(t))=\frac{d}{d t} \ell_{\dot{x}}(\hat{x}(t), \dot{\hat{x}}(t))-\delta \ell_{\dot{x}}(\hat{x}(t), \dot{\hat{x}}(t)) .
$$

But this is equivalent to the assertion: $\hat{y}:=\hat{x} \circ \gamma^{-1}$ satisfies the following Euler-Lagrange equation:

$$
\forall s \in\left[-\delta^{-1}, 0\right], \frac{d}{d s} \mathcal{L}_{\dot{y}}(s, \hat{y}(s), \dot{\hat{y}}(s))=\mathcal{L}_{y}(s, \hat{y}(s), \dot{\hat{y}}(s)) .
$$

Since $(\mathrm{C}, \hat{x})$ is verified, $\hat{x}(t)$ satisfies the following strengthened Legendre condition: $\ell_{\dot{x} \dot{x}}(\hat{x}(t), \dot{\hat{x}}(t))$ is negative definite, for all $t \in \mathbb{R}_{+}$. Consequently $\hat{y}(s)$ satisfies the following Strengthened Legendre condition: $\mathcal{L}_{\dot{y} \dot{y}}(s, \hat{y}(s), \dot{\hat{y}}(s))$ is negative definite, for all $s \in\left[-\delta^{-1}, 0\right]$. On the other hand the Jacobi equation around $\hat{x}$ goes like (removing the argument $t$ ):

$$
\ell_{x x}(\hat{x}, \dot{\hat{x}}) h+\ell_{x \dot{x}}(\hat{x}, \dot{\hat{x}}) \dot{h}=\frac{d}{d t}\left[\ell_{\dot{x} x}(\hat{x}, \dot{\hat{x}}) h+\ell_{\dot{x} \dot{x}}(\hat{x}, \dot{\hat{x}}) \dot{h}\right]-\delta\left(\ell_{\dot{x} x}(\hat{x}, \dot{\hat{x}}) h+\ell_{\dot{x} \dot{x}}(\hat{x}, \dot{\hat{x}}) \dot{h}\right) .
$$

We denote it by $(J a c)_{\hat{x}}$. It is easy to see that $h$ is a solution of $(J a c)_{\hat{x}}$ if and only if $k=h \circ \gamma^{-1}$ is a solution of $(J a c) \hat{y}$ which is (removing the argument $s$ ):

$$
\mathcal{L}_{y y}(s, \hat{y}, \dot{\hat{y}}) k+\mathcal{L}_{y \dot{y}}(s, \hat{y}, \dot{\hat{y}}) \dot{k}=\frac{d}{d s}\left[\mathcal{L}_{\dot{y} y}(s, \hat{y}, \dot{\hat{y}}) k+\mathcal{L}_{\dot{y} \dot{y}}(s, \hat{y}, \dot{\hat{y}}) \dot{k}\right] .
$$

Knowing that $\left(\mathrm{D}^{\prime \prime}, \hat{x}\right)$ is satisfied, we assert that there does not exist a conjugate point to $-\delta^{-1}$ on $\left(-\delta^{-1}, 0\right]$ along $\hat{y}$. Hence a classical theorem in finite-horizon calculus of variations problems [15] can be applied to obtain that $\hat{y}$ is embeddable in a Mayer field $\tilde{f}: \tilde{N} \longrightarrow \tilde{M}$, such that $\tilde{f}(s, \alpha)=(s, \tilde{\Phi}(s, \alpha))$ and whose slope is $\tilde{u}: \tilde{M} \longrightarrow \mathbb{R}^{n}$.

Now since $\left(\mathrm{B}^{\prime}, \hat{x}\right)$ is satisfied, we obtain the following condition: there exists $c_{1}>0$ such that for every $(s, y, v, w)$ verifying $s \in\left[-\delta^{-1}, 0\right],(y, v) \in \Omega,(y, w) \in \Omega$, and for all $s \in\left[-\delta^{-1}, 0\right],\|y-\hat{y}(s)\|+\|v-\dot{\hat{y}}(s)\|<c_{1}$, we have $\mathcal{L}(s, y, w)-\mathcal{L}(s, y, v)-\mathcal{L}_{\dot{y}}(s, y, v)(w-v) \leq 0$.

Since $\left(\mathcal{F}_{\delta}\right)$ is a free-endpoint problem, in order to apply a classical finite-horizon theorem that ensures that $\hat{y}$ is a local strong solution of $\left(\mathcal{F}_{\delta}\right)$ in the usual sense, we need to have the following transversality condition fulfilled: $\mathcal{L}_{\dot{y}}\left(0, y(0), \tilde{u}(0, y(0))=0\right.$. But this is satisfied since $\mathcal{L}_{\dot{y}}(0, y(0), \dot{y}(0))=\ell_{\dot{x}}(y(0),-\delta .0 . \dot{y}(0))(-\delta .0)=0$.

Therefore there exists $c>0$ such that, for every $y \in C^{1}\left(\left[-\delta^{-1}, 0\right]\right.$ verifying $y\left(-\delta^{-1}\right)=\eta$, we have $\left[\|y-\hat{y}\|_{\infty}\right.$ $<c]$ implies $\mathcal{J}_{\delta}(y) \leq \mathcal{J}_{\delta}(\hat{y})$.

It is now easy to show that, for every $z \in N C_{\mathcal{O}}^{1}$ verifying $z(0)=\eta$, we have $\left[\forall t \in \mathbb{R}_{+},\|z(t)-\hat{x}(t)\|<c\right]$ implies $J(z) \leq J(\hat{x})$.

We finally show that, for every $z \in \operatorname{Adm}(\eta),\left[\forall t \in \mathbb{R}_{+},\|z(t)-\hat{x}(t)\|<c\right]$ implies $J(z) \leq J(\hat{x})$. Proceeding as in [3], let $z \in \operatorname{Adm}(\eta)$ verify for every $t \in \mathbb{R}_{+},[\|z(t)-\hat{x}(t)\|<r]$. For $T>0$, set $z_{T}(t):=z(t)$ if $t \in[0, T]$ and $z_{T}(t):=z(T)$ if $t \in[T,+\infty]$. So $\dot{z}_{T}(t)=0$ if $t>T$. Therefore $\dot{z}_{T}(t) e^{\delta t}=0$ if $t>T$, and $\lim _{t \rightarrow+\infty} \dot{z}_{T}(t) e^{\delta t}=0$.

So $z \in N C_{\mathcal{O}}^{1} \cap P C^{1}\left(\mathbb{R}_{+}, \mathbb{R}^{n}\right)$ and verifies $z(0)=\eta$ and for every $t \in \mathbb{R}_{+}\|z(t)-\hat{x}(t)\|<r$. So $J_{T}\left(z_{T}\right)$ $\geq \int_{0}^{T} L(t, z(t), \dot{z}(t)) d t$ since $\ell \geq 0$. Therefore $J(\hat{x}) \geq J_{T}\left(z_{T}\right) \geq \int_{0}^{T} L(t, z(t), \dot{z}(t)) d t$ and $J(\hat{x}) \geq J(z)$.

\section{Sufficient CONDitions Via AUXiliary FunCtions}

In the study of stability for ordinary differential equations, two families of methods exist: linearization and Lyapounov's direct methods (using auxiliary functions). 
Similarly, in the study of sufficient conditions for calculus of variations problems, starting with a solution of the Euler-Lagrange equation, aside the usual methods related to the Jacobi equation (that is the linearized Euler-Lagrange equation), Nehari [21] provides another method using auxiliary functions.

In this section we extend his approach to infinite-horizon problems.

Theorem 7. Under (3), let $\hat{x} \in C_{\mathcal{O}}^{1}$ satisfy $\hat{x}(0)=\eta$. If the following conditions are fulfilled:

- $\hat{x}$ satisfies the Euler-Lagrange equation;

- there exists a function $G=G(t, x)$ such that for all $t \in \mathbb{R}_{+}, L-G_{x} \dot{x}-G_{t}$ is concave with respect to $(x, \dot{x})$;

- $\limsup _{T \rightarrow+\infty}\left\{\left[L_{\dot{x}}(T, \hat{x}(T), \dot{\hat{x}}(T))-G_{x}(T, \hat{x}(T))\right](x(T)-\hat{x}(T))+G(T, x(T))-G(T, \hat{x}(T))\right\} \leq 0$, for all $x \in C_{\mathcal{O}}^{1}$ $T \rightarrow+\infty$
such that $\hat{x}(0)=\eta$

then $\hat{x}$ is (III)-optimal.

Proof. Following Nehari [21], we set

$$
\mathcal{F}(L(t, x, \dot{x})):=L(t, x, \dot{x})-L(t, \hat{x}, \dot{\hat{x}})-L_{x}(t, \hat{x}, \dot{\hat{x}})(x-\hat{x})-L_{\dot{x}}(t, \hat{x}, \dot{\hat{x}})(\dot{x}-\dot{\hat{x}}) .
$$

So

$$
\begin{gathered}
J_{T}(x)-J_{T}(\hat{x}) \\
=\int_{0}^{T} \mathcal{F}\left(L(t, x(t), \dot{x}(t))-\frac{d G(t, x(t))}{d t}\right) d t+L_{\dot{x}}(T, \hat{x}(T), \dot{\hat{x}}(T))(x(T)-\hat{x}(T)) \\
-G_{x}(T, \hat{x}(T))(x(T)-\hat{x}(T))+G(T, x(T))-G(T, \hat{x}(T)) .
\end{gathered}
$$

\section{Appendix: Extremal FieldS}

Let $M$ be an open simply connected subset of $\mathbb{R} \times \mathbb{R}^{n}, N$ be an open subset of $\mathbb{R} \times \mathbb{R}^{n}$, and $\phi: N \longrightarrow \mathbb{R}^{n}$ be a mapping such that for every $\alpha \in \operatorname{proj}_{2}(N), \phi(., \alpha)$ is an extremal.

The mapping $f: N \longrightarrow M$, defined by $f(t, \alpha):=(t, \phi(t, \alpha))$, is called an extremal field when $f$ is a $C^{1}$-diffeomorphism from $N$ onto $M$ and the partial differential $f_{t}$ is of class $C^{1}$ on $N$.

The slope $u$ of $f$ is the $C^{1}$ mapping $u: M \longrightarrow \mathbb{R}^{n}$ defined by $u:=\phi_{t} \circ f^{-1}$.

A $C^{1}$-curve $x$, defined on an interval $\mathcal{I} \subset \mathbb{R}$, is called embeddable in the extremal field $f$ when, for every $t \in \mathcal{I}$, $(t, x(t)) \in M$ and $\dot{x}(t)=u(t, x(t))$.

An extremal field $f: N \longrightarrow M$, is called a Mayer field when its slope $u: M \longrightarrow \mathbb{R}^{n}$, satisfies the following equations, for every $(t, x) \in M$, for every $i \in\{1, \ldots, n\}$, for every $k \in\{1, \ldots, n\}$,

$$
\frac{\partial}{\partial x^{k}} L_{\dot{x}^{i}}(t, x, u(t, x))=\frac{\partial}{\partial x^{i}} L_{\dot{x}^{k}}(t, x, u(t, x)) .
$$

When $n=1$, these equations are automatically satisfied, and so an extremal field is always a Mayer field.

Proposition A1. Let $N_{0}$ be an open subset of $\mathbb{R} \times \mathbb{R}^{n}$, such that $\operatorname{proj}_{1}\left(N_{0}\right)$ is of the form $(\underline{t},+\infty)$.

Let $\phi: N_{0} \longrightarrow \mathbb{R}^{n}$ be a mapping which satisfies the following conditions:

(i) $\phi \in C^{1}\left(N_{0}, \mathbb{R}^{n}\right)$, and $\phi_{t} \in C^{1}\left(N_{0}, \mathbb{R}^{n}\right)$.

(ii) For each $\alpha \in \operatorname{proj}_{2}\left(N_{0}\right), \phi(., \alpha)$ is an extremal on $(\underline{t},+\infty)$.

(iii) There exists $\hat{\alpha} \in \operatorname{proj}_{2}\left(N_{0}\right)$ such that, for every $t \in \operatorname{proj}_{1}\left(N_{0}\right)$, we have $(t, \hat{\alpha}) \in N_{0}$ and $\operatorname{det} \phi_{\alpha}(t, \hat{\alpha}) \neq 0$. Then, $\hat{x}():.=\phi(., \hat{\alpha})$ is embeddable in an extremal field. 
Proof. We define the mapping $g: N_{0} \longrightarrow \mathbb{R} \times \mathbb{R}^{n}$ by taking $g(t, \alpha):=(t, \phi(t, \alpha))$. Then using assumptions (i) and (iii), we note that $g$ is of class $C^{1}$ and $g^{\prime}(t, \hat{\alpha}) \in G L\left(\mathbb{R} \times \mathbb{R}^{n}\right)$ for every $t \in \operatorname{proj}_{1}\left(N_{0}\right)$.

Arbitrarily we take $t_{0}<t_{1}$ such that $\left[t_{0}, t_{1}\right] \subset(\underline{t},+\infty)$. Then by using the local inversion theorem and the compactness of $\left[t_{0}, t_{1}\right] \times\{\hat{\alpha}\}$, there exists $\rho_{t_{0}, t_{1}}>0$ such that $\left[t_{0}, t_{1}\right] \times B\left(\hat{\alpha}, \rho_{t_{0}, t_{1}}\right) \subset N_{0}$, and the restriction of $g$ to $\left[t_{0}, t_{1}\right] \times B\left(\hat{\alpha}, \rho_{t_{0}, t_{1}}\right)$ is one-to-one.

To simplify the notation, we set $\rho_{n}:=\rho_{n, n+1}$ for every $n \geq a$. We divide $(\underline{t},+\infty)$ in intervals of length equal to one, starting from $a:=\min \{k \in \mathbb{Z}: k \geq \underline{t}\}$, and we define the function $\rho:[a,+\infty) \longrightarrow(0,+\infty)$ as follows:

$$
\rho(t):=\left\{\begin{array}{cl}
\rho_{a} & \text { if } \quad t \in[a, a+1) \\
\min \left\{\rho_{n-1}, \rho_{n}\right\} & \text { if } \quad t \in[n, n+1), n>a .
\end{array}\right.
$$

We note that $\rho$ is lower semicontinuous. The Dowker theorem ([10], p. 171), implies the existence of a function $\Gamma \in C^{0}([a,+\infty),(0,+\infty))$ such that for every $t \in[a,+\infty), \rho(t) \geq \Gamma(t)>0$.

Now we set

$$
N:=\{(t, \alpha): t \in(a,+\infty),\|\alpha-\hat{\alpha}\|<\Gamma(t)\}
$$

We note that $N$ is open in $\mathbb{R} \times \mathbb{R}^{n}$. To show that $N$ is pathwise connected, for each $\left(\tau_{0}, \alpha_{0}\right),\left(\tau_{1}, \alpha_{1}\right) \in N$, we consider the path obtained by joining the segment that links $\left(\tau_{0}, \alpha_{0}\right)$ to $\left(\tau_{0}, \hat{\alpha}\right)$, the segment that links $\left(\tau_{0}, \hat{\alpha}\right)$ to $\left(\tau_{1}, \hat{\alpha}\right)$, and the segment that links $\left(\tau_{1}, \hat{\alpha}\right)$ to $\left(\tau_{1}, \alpha_{1}\right)$. After that, to prove that $N$ is simply connected, i.e. to prove that each loop $\sigma(\theta)=\left(\sigma^{1}(\theta), \sigma^{2}(\theta)\right)$ with values in $N$ is homotopic to a point, it is easy to show that $\sigma$ is homotopic to the loop $\gamma(\theta):=\left(\sigma^{1}(\theta), \hat{\alpha}\right)$, and that $\gamma$ is homotopic to the point $\left(\sigma^{1}(0), \hat{\alpha}\right)$.

For each $(t, \alpha) \in N$, there exists $n \in \mathbb{Z}, n \geq a$, such that $t \in[n, n+1)$ and $\alpha \in B\left(\hat{\alpha}, \rho_{n}\right)$, therefore $(t, \alpha) \in N_{0}$. And so, we have $N \subset N_{0}$.

We set $M:=g(N) \subset g\left(N_{0}\right)$, and we denote by $f$ the restriction of $g$ to $N$.

Let $\left(t^{\prime}, \alpha^{\prime}\right),\left(t^{\prime \prime}, \alpha^{\prime \prime}\right) \in N$ such that $f\left(t^{\prime}, \alpha^{\prime}\right)=f\left(t^{\prime \prime}, \alpha^{\prime \prime}\right)$. Then we have $t^{\prime}=t^{\prime \prime}$, and $\alpha^{\prime}, \alpha^{\prime \prime} \in B(\hat{\alpha}, \Gamma(t))$ $\subset B\left(\hat{\alpha}, \rho\left(t^{\prime}\right)\right)$. Therefore there exists $n \in(a,+\infty) \cap \mathbb{Z}$ such that $t^{\prime} \in[n, n+1)$, and so $\alpha^{\prime}, \alpha^{\prime \prime} \in B\left(\hat{\alpha}, \rho_{n}\right)$. Consequently we have $\left(t^{\prime}, \alpha^{\prime}\right),\left(t^{\prime}, \alpha^{\prime \prime}\right) \in[n, n+1) \times B\left(\hat{\alpha}, \rho_{n}\right)$, and since $g$ is one-to-one on this set, we have $\left(t^{\prime}, \alpha^{\prime}\right)=\left(t^{\prime \prime}, \alpha^{\prime \prime}\right)$. And so, we have proved that $f$ is one-to-one on $N$.

Moreover $f$ is of class $C^{1}$ and, for each $(t, \alpha) \in N, f^{\prime}(t, \alpha)=g^{\prime}(t, \alpha) \in G L\left(\mathbb{R} \times \mathbb{R}^{n}\right)$, therefore ([8], p. 56, Cor. 4.2.2), $M=f(N)$ is open and the restriction $f: N \longrightarrow M$ is a $C^{1}$-diffeomorphism. $M$ is pathwise connected since $f$ is continuous, and the associated map (between the first homotopy groups) $f_{*}: \Pi_{1}(N) \longmapsto \Pi_{1}(M)$ is a bijection since $f$ is a homeomorphism [15], and so since $\Pi_{1}(N)$ is trivial, $\Pi_{1}(M)$ is trivial, i.e. $M$ is simply connected.

Proposition A2. Under (3) and (4), let $\hat{x} \in C_{\mathcal{O}}^{1}$. If $(A, \hat{x}),(C, \hat{x})$, and (D', $\left.\hat{x}\right)$ are fulfilled, then $\hat{x}$ is embeddable in a Mayer field.

Proof. The condition $(\mathrm{C}, \hat{x})$ implies that there exists $t_{2} \in\left[t_{0}, 0\right)$ and there exists $e \in C^{0}\left(\left[t_{2},+\infty\right),(0,+\infty)\right)$ such that, for every $(t, x, v) \in \mathcal{O}$,

$$
\left(t \in\left[t_{2},+\infty\right),\|x-\hat{x}(t)\|<e(t),\|v-\dot{\hat{x}}(t)\|<e(t)\right) \Longrightarrow L_{\dot{x} \dot{x}}(t, x, v)<0
$$

We introduce the set $\hat{\mathcal{O}}:=\left\{(t, x, v) \in \mathcal{O}: t \in\left[t_{2},+\infty\right),\|x-\hat{x}(t)\|<e(t),\|v-\dot{\hat{x}}(t)\|<e(t)\right\}$. We see that $\hat{\mathcal{O}}$ is open, and for each $(t, x)$, if $\hat{\mathcal{O}}(t, x):=\left\{v \in \mathbb{R}^{n}:(t, x, v) \in \hat{\mathcal{O}}\right\}$ is nonempty, then it is convex as an intersection of two convex sets.

This will guarantee that there exists a domain $\hat{\mathcal{D}} \subset\left(t_{2},+\infty\right) \times \mathbb{R}^{n} \times \mathbb{R}^{n}$ such that $H \in C^{2}(\hat{\mathcal{D}}, \mathbb{R})$.

By setting $\hat{p}(t):=-L_{\dot{x}}(t, \hat{x}(t), \dot{\hat{x}}(t))$, we have $(\hat{x}, \hat{p})$ is a solution of $(5)$ on an interval $(-\delta,+\infty)$, with $\delta>0$. We fix $t^{*} \in\left(\min \left\{-\delta, t_{2}\right\}, 0\right)$, we set $x^{*}:=\hat{x}\left(t^{*}\right)$, we define the set $\mathcal{T}:=\left\{(t, \alpha):\left(t, t^{*}, x^{*}, \alpha\right) \in \operatorname{dom}(\mathrm{X}, \mathrm{P})\right\}$, we 
define the mappings $\phi(t, \alpha):=\mathrm{X}\left(t, t^{*}, x^{*}, \alpha\right)$ and $\psi(t, \alpha):=\mathrm{P}\left(t, t^{*}, x^{*}, \alpha\right)$, we consider the set $\mathcal{A}:=\left\{\alpha \in \mathbb{R}^{n}\right.$ : $\left.\forall t \in\left(\frac{t^{*}}{2},+\infty\right),(t, \alpha) \in \mathcal{T},(t, \phi(t, \alpha), \psi(t, \alpha)) \in \hat{\mathcal{D}}\right\}$, and we set $N_{0}:=\left(\frac{t^{*}}{2},+\infty\right) \times \mathcal{A}$.

Then using $\left(\mathrm{D}^{\prime}, \hat{x}\right)$, for every $t \in\left(\frac{t^{*}}{2},+\infty\right)$, we have $\operatorname{det} \phi_{\alpha}(t, \hat{\alpha}) \neq 0$.

Since $(\mathrm{X}, \mathrm{P})$ is the flow of the ODE (5), by using the dependence theorems of ODE [1], we obtain that $\phi_{\alpha}$, $\phi_{t}, \phi_{t \alpha}, \phi_{\alpha \alpha}$ are continuous on $N_{0}$.

And we can use Proposition 1 to assert that $\hat{x}$ is embeddable in an extremal field. To show that the extremal field is a Mayer field, the reasoning is similar to the one provided in [14], p. 323.

Lemma A1. Under (3), let $\hat{x} \in C_{\mathcal{O}}^{1}$ be embeddable in an extremal field $f: N \longrightarrow M$ whose slope is $u: M \longrightarrow \mathbb{R}^{n}$. If $(E, \hat{x})$ holds, then there exists $r_{1} \in B C^{0}\left(\mathbb{R}_{+},(0,+\infty)\right)$ such that, for every $y: \mathbb{R}_{+} \rightarrow \mathbb{R}^{n}$ verifying, for every $t \in \mathbb{R}_{+},(t, y(t)) \in N,(t, y(t), u(t, y(t))) \in \mathcal{O}$ and $\|y(t)-\hat{x}(t)\|<r_{1}(t)$, we have: $\lim _{t \rightarrow+\infty} L_{\dot{x}}(t, y(t), u(t, y(t)))=0$.

Proof. We set $g(t, y):=L_{\dot{x}}(t, y, u(t, y))$. For each $n \in \mathbb{N},\{(t, \hat{x}(t)): t \in[n, n+1]\}$ is compact and $g$ is continuous, so the Heine-Schwartz theorem ([26], p. 355), implies that there exists $\beta_{n}>0$ such that, for every $y: \mathbb{R}_{+} \rightarrow \mathbb{R}^{n}$ verifying, for every $t \in \mathbb{R}_{+},(t, y(t)) \in N,(t, y(t), u(t, y(t))) \in \mathcal{O}$, we have:

$$
\begin{gathered}
\left(\forall t \in[n, n+1],\|y(t)-\hat{x}(t)\| \leq \beta_{n}\right) \Longrightarrow \\
\left(\forall t \in[n, n+1],\|g(t, y(t))-g(t, \hat{x}(t))\| \leq \frac{1}{n+1}\right) .
\end{gathered}
$$

We define the function $\beta: \mathbb{R}_{+} \longrightarrow(0,+\infty)$ as follows:

$$
\beta(t):=\min \left\{\beta_{k}: 0 \leq k \leq n+1\right\} \text { when } t \in[n, n+1) .
$$

Hence $\beta$ is lower semicontinuous. Then the Dowker theorem ensures the existence of a function $r_{1} \in B C^{0}\left(\mathbb{R}_{+}\right.$, $(0,+\infty))$ such that $\beta(t) \geq r_{1}(t)>0$ for every $t \in \mathbb{R}_{+}$.

If $y \in C^{1}\left(\mathbb{R}_{+}, \mathbb{R}^{n}\right)$ is such that, for every $t \in \mathbb{R}_{+},(t, y(t)) \in N,(t, y(t), u(t, y(t))) \in \mathcal{O},\|y(t)-\hat{x}(t)\|<r_{1}(t)$, then for each $t \in(0,+\infty)$, there exists $n \in \mathbb{N}$ verifying $t \in[n, n+1)$, and so we have $\|y(t)-\hat{x}(t)\| \leq \beta_{n}$ that implies $\|g(t, y(t))-g(t, \hat{x}(t))\| \leq \frac{1}{n+1}<\frac{1}{t}$, therefore we have: $\|g(t, y(t))\| \leq\|g(t, \hat{x}(t))\|+\frac{1}{t}$. And since $\lim _{t \rightarrow+\infty} g(t, \hat{x}(t))=0$, we obtain $\lim _{t \rightarrow+\infty} g(t, y(t))=0$.

When we consider an extremal field $f: N \longrightarrow M$ whose slope is $u: M \longrightarrow \mathbb{R}^{n}$, for each curve $x \in C_{\mathcal{O}}^{1}$ such that, for every $t \in \mathbb{R}_{+},(t, x(t), u(t, x(t))) \in M$, for each $T \in(0,+\infty)$, we denote by $[x]_{T}$ the parametrized curve $t \longmapsto(t, x(t))$, from $[0, T]$ in $\mathbb{R}^{N}$, and we define the following line integral:

$$
J_{T}^{*}(x):=\int_{[x]_{T}}\left[L(t, x, u(t, x))-L_{\dot{x}}(t, x, u(t, x)) \cdot u(t, x)\right] d t+\sum_{k=1}^{n} L_{\dot{x}^{k}}(t, x, u(t, x)) d x_{k} .
$$

Under (3), for every $(t, x) \in M$, the following equality holds:

$$
\begin{aligned}
L_{x}(t, x, u(t, x))= & L_{\dot{x} t}(t, x, u(t, x))+L_{\dot{x} x}(t, x, u(t, x)) \cdot u(t, x) \\
& +L_{\dot{x} \dot{x}}(t, x, u(t, x)) \cdot\left[u_{t}(t, x)+u_{x}(t, x) \cdot u(t, x)\right] .
\end{aligned}
$$

Proposition A3. Under (3), let $\hat{x} \in C_{\mathcal{O}}^{1}$ satisfy $\hat{x}(0)=\eta$. If $\hat{x}$ is embeddable in a Mayer field $f: N \longrightarrow M$ whose slope is $u: M \longrightarrow \mathbb{R}^{n}$, and if $(E, \hat{x})$ is satisfied, then there exists $r_{1} \in B C^{0}\left(\mathbb{R}_{+},(0,+\infty)\right)$ such that, for 
every $x \in C_{\mathcal{O}}^{1}$ verifying $x(0)=\eta$, and for every $t \in \mathbb{R}_{+},(t, x(t)) \in M,\|x(t)-\hat{x}(t)\|<r_{1}(t)$, the two following assertions hold:

(i) $\lim _{T \rightarrow+\infty}\left(J_{T}^{*}(x)-J_{T}^{*}(\hat{x})\right)=0$.

(ii) $\limsup _{T \rightarrow+\infty}\left(J_{T}(x)-J_{T}(\hat{x})\right)=\limsup _{T \rightarrow+\infty} \int_{0}^{T} \mathcal{E}(t, x(t), u(t, x(t)), \dot{x}(t)) d t$.

Proof. Since $f$ is a Mayer field, by using (10), the integrand of $J_{T}^{*}(x)$ satisfies the conditions which ensure that $J_{T}^{*}(x)$ is independent of the path of integration and which permit us to define $F: M \longrightarrow \mathbb{R}$ such that $F_{t}=L-L_{\dot{x}} \cdot u$ and $F_{x}=L_{\dot{x}}[8]$, the arguments of $F$ and $u$ being $(t, x)$ and those $L$ and $L_{\dot{x}}$ being $(t, x, u(t, x))$.

Hence we have

$$
J_{T}^{*}(x)-J_{T}^{*}(\hat{x})=F(T, x(T))-F(T, \hat{x}(T)) .
$$

So by using the mean-value theorem we obtain

$$
\left|J_{T}^{*}(x)-J_{T}^{*}(\hat{x})\right| \leq \sup _{\gamma_{T} \in(x(T), \hat{x}(T))}\left\|L_{\dot{x}}\left(T, \gamma_{T}, u\left(T, \gamma_{T}\right)\right)\right\| \cdot\|x(T)-\hat{x}(T)\| .
$$

If $r_{1}$ denotes the function provided by Lemma A1, if now we assume that $\|x(t)-\hat{x}(t)\|<r_{1}(t)$, for every $t \in \mathbb{R}_{+}$, then we can assert that $\lim _{T \rightarrow+\infty} L_{\dot{x}}\left(T, \gamma_{T}, u\left(T, \gamma_{T}\right)\right)=0$ which implies (i).

To prove (ii) it is sufficient to note that $J_{T}(\hat{x})=J_{T}^{*}(\hat{x}), J_{T}(x)-J_{T}(\hat{x})=J_{T}(x)-J_{T}^{*}(x)+J_{T}^{*}(x)-J_{T}^{*}(\hat{x})$ and to use (i).

\section{REFERENCES}

[1] V.M. Alexeev, V.M. Tikhomirov and S.V. Fomin, Commande optimale, French translation. Mir, Moscow (1982).

[2] K.J. Arrow, Applications of Control Theory to Economic Growth. Math. of the Decision Sciences, edited by G.B. Dantzig and A.F. Veinott Jr. (1968).

[3] J. Blot and P. Cartigny, Optimality in Infinite-Horizon Problems under Signs Conditions. J. Optim. Theory Appl. (to appear).

[4] J. Blot and N. Hayek, Second-Order Necessary Conditions for the Infinite-Horizon Variational Problems. Math. Oper. Res. 21 (1996) 979-990.

[5] J. Blot and Ph. Michel, First-Order Necessary Conditions for the Infinite-Horizon Variational Problems. J. Optim. Theory Appl. 88 (1996) 339-364.

[6] N. Bourbaki, Fonctions d'une variable réelle. Hermann, Paris (1976).

[7] D.A. Carlson, A.B. Haurie and A. Leizarowitz, Infinite Horizon Optimal Control, Deterministic and Stochastic Systems, Second Edition. Springer-Verlag, Berlin (1991).

[8] H. Cartan, Calcul Différentiel. Hermann, Paris (1967).

[9] L. Cesari, Optimization Theory and Applications: Problems with Ordinary Differential Equations. Springer-Verlag, New York (1983).

[10] J. Dugundji, Topology. Allyn and Bacon, Boston (1966).

[11] G.E. Ewing, Calculus of Variations, with Applications. Dover Pub. Inc., New York (1985).

[12] W.H. Fleming and R. Rishel, Deterministic and Stochastic Optimal Control. Springer-Verlag, New York (1975).

[13] W.H. Fleming and H.M. Soner, Controlled Markov Processes and Viscosity Solutions. Springer-Verlag, New York (1993).

[14] M. Giaquinta and S. Hildebrandt, Calculus of Variations I. Springer-Verlag, Berlin (1996).

[15] C. Godbillon, Éléments de topologie algébrique. Hermann, Paris (1971).

[16] R.F. Hartl, S.P. Sethi and R.G. Vickson, A Survey of the Maximum Principles for Optimal Control Problems with State Constraints. SIAM Rev. 37 (1995) 181-218.

[17] M.H. Hestenes, Calculus of Variations and Optimal Control Theory. Robert E. Krieger Publ. Comp., Huntington, N.Y. (1980).

[18] G. Leitman and H. Stalford, A Sufficiency Theorem for Optimal Control. J. Optim. Theory Appl. VIII (1971) 169-174.

[19] D. Leonard and N.V. Long, Optimal Control Theory and Static Optimization in Economics. Cambridge University Press, New York (1992).

[20] O.L. Mangasarian, Sufficient Conditions for the Optimal Control of Nonlinear Systems. SIAM J. Control IV (1966) 139-152.

[21] Z. Nehari, Sufficient Conditions in the Calculus of Variations and in the Theory of Optimal Control. Proc. Amer. Math. Soc. 39 (1973) 535-539. 
[22] L. Pontryagin, V. Boltyanskii, R. Gramkrelidze and E. Mitchenko, Théorie Mathématique des Processus Optimaux, French Edition. Mir, Moscow (1974).

[23] H. Sagan, Introduction to the Calculus of Variations. McGraw-Hill, New York (1969).

[24] Th. Sargent, Macroeconomic Theory, Second Edition. Academic Press, New York (1986).

[25] A. Seierstad and K. Sydsaeter, Sufficient Conditions in Optimal Control Theory, Internat. Econom. Rev. 18 (1977).

[26] L. Schwartz, Cours d'Analyse de l'École Polytechnique, Tome 1. Hermann, Paris (1967).

[27] L. Schwartz, Topologie Générale et Analyse Fonctionnelle. Hermann, Paris (1970).

[28] G. Sorger, Sufficient Conditions for Nonconvex Control Problems with State Constraints. J. Optim. Theory Appl. 62 (1989) 289-310.

[29] J.L. Troutman, Variational Calculus with Elementary Convexity. Springer-Verlag, New York (1983).

[30] V. Zeidan, First and Second Order Sufficient Conditions for Optimal Control and Calculus of Variations. Appl. Math. Optim. 11 (1984) 209-226.

[31] A.J. Zaslavski, Existence and Structure of Optimal Solutions of Variational Problems, Recent Developments in Optimization Theory and Nonlinear Analysis, edited by Y. Censor and S. Reich. Amer. Math. Soc. Providence, Rhode Island (1997) $247-278$. 